\title{
The mismatch repair system (mutS and mutL) in Acinetobacter baylyi ADP1
}

Hua Zhou ${ }^{1 \dagger}$, Linyue Zhang ${ }^{2,3 \dagger}$, Qingye $X^{2,3}$, Linghong Zhang ${ }^{2,3}$, Yunsong $\mathrm{Yu}^{2,3^{*}}$ and Xiaoting Hua ${ }^{2,3^{*}}$ (D)

\begin{abstract}
Background: Acinetobacter baylyi ADP1 is an ideal bacterial strain for high-throughput genetic analysis as the bacterium is naturally transformable. Thus, ADP1 can be used to investigate DNA mismatch repair, a mechanism for repairing mismatched bases. We used the mutS deletion mutant (XH439) and mutL deletion mutant (XH440), and constructed a mutS mutL double deletion mutant (XH441) to investigate the role of the mismatch repair system in A. baylyi.

Results: We determined the survival rates after UV irradiation and measured the mutation frequencies, rates and spectra of wild-type ADP1 and mutSL mutant via rifampin resistance assay (Rif ${ }^{R}$ assay) and experimental evolution. In addition, transformation efficiencies of genomic DNA in ADP1 and its three mutants were determined. Lastly, the relative growth rates of the wild type strain, three constructed deletion mutants, as well as the rifampin resistant mutants obtained from Rif ${ }^{\mathrm{R}}$ assays, were measured. All three mutants had higher survival rates after UV irradiation than wild type, especially the double deletion mutant. Three mutants showed higher mutation frequencies than ADP1 and favored transition mutations in if $^{R}$ assay. All three mutants showed increased mutation rates in the experimental evolution. However, only XH439 and XH441 had higher mutation rates than the wild type strain in Rif $^{\mathrm{R}}$ assay. XH441 showed higher transformation efficiency than XH438 when donor DNA harbored transition mutations. All three mutants showed higher growth rates than wild-type, and these four strains displayed higher growth rates than almost all their rpoB mutants. The growth rate results showed different amino acid mutations in $r p o B$ resulted in different extents of reduction in the fitness of rifampin resistant mutants. However, the fitness cost brought by the same mutation did not vary with strain background.

Conclusions: We demonstrated that inactivation of both mutS and mutL increased the mutation rates and frequencies in A. baylyi, which would contribute to the evolution and acquirement of rifampicin resistance. The mutS deletion is also implicated in increased mutation rates and frequencies, suggesting that MutL may be activated even in the absence of mutS. The correlation between fitness cost and rifampin resistance mutations in $A$. baylyi is firstly established.
\end{abstract}

Keywords: Acinetobacter baylyi, mutS, mutL, Mutation, Antibiotic resistance, Resistance evolution

\section{Background}

Acinetobacter baylyi ADP1 is a Gram-negative, nonpathogenic soil bacterium [1]. It is considered as an ideal organism for high-throughput genetic analysis, metabolic engineering and synthetic biology because it possesses a natural transformation system $[2,3]$.

\footnotetext{
* Correspondence: yvys119@zju.edu.cn; XiaotingHua@zju.edu.cn

${ }^{\dagger}$ Hua Zhou and Linyue Zhang are the authors contributed equally to this work and should be considered as co-first author.

${ }^{2}$ Department of Infectious Diseases, Sir Run Run Shaw Hospital, Zhejiang

University School of Medicine, Hangzhou, China

Full list of author information is available at the end of the article
}

Mutagenized DNA can be transformed into ADP1 to make sequence variations at specific sites in the genomic DNA [4]. Previously, the genome of A. baylyi ADP1 was sequenced [5], and a nearly complete collection of ADP1 mutants was constructed [2]. We used two mutants from this collection for further study.

DNA mismatch repair (MMR) is a DNA repair pathway focusing on repairing mismatched bases [6]. Mutations in DNA mismatch repair proteins (mutS and mutL) could confer hypermutator phenotypes and might facilitate the emergence of mutational antibiotic resistance in bacteria [7]. The MMR system was originally

(c) The Author(s). 2020 Open Access This article is distributed under the terms of the Creative Commons Attribution 4.0 International License (http://creativecommons.org/licenses/by/4.0/), which permits unrestricted use, distribution, and 
described in Streptococcus pneumoniae in 1962 [8, 9]. Currently, the best-characterized MMR systems are from E. coli and Bacillus subtilis [10]. The homologues of MutS exist in all prokaryotes, with the exception of Actinobacteria, Mollicutes and part of the Archaea [11]. Bacteria of the Actinobacteria Phylum and Archaea encode a non-canonical MMR system (NucS/endoMS) [12]. For Mollicutes, it was reported that the histone-like protein HU (Hup2) in M. gallisepticum might play it role in mismatch repair [13].

A previous study showed that strains lacking $m u t S$ exhibited increased spontaneous mutation frequencies in $A$. baylyi. Inactivating mutS also affected the transformation frequencies with divergent donor sequences with showing specificity for transition and frameshift mismatches in a marker replacement assay [14]. However, the influence of single mutL deletion and mutS mutL double deletion in ADP1 haven't been studied yet. In this study, we constructed a mutS mutL double mutant ( $\Delta m u t S \Delta m u t L: \mathrm{XH} 441)$, and we also adapted single deletion mutants ( $\Delta m u t S:$ XH439, $\Delta m u t L:$ XH440) to provide a thorough understanding of the mismatch repair system in A. baylyi. We determined the survival rates after UV irradiation, and investigated the mutation frequencies, rates and spectrums of wild-type ADP1
(XH438), $\mathrm{XH} 439, \mathrm{XH440,}$ as well as $\mathrm{XH} 441$ via antibiotic rifampin resistance assay ( $\operatorname{Rif}^{\mathrm{R}}$ assay) and experimental evolution. In addition, transformation efficiencies of genomic DNA in ADP1 and its three mutants were determined. Lastly, the relative growth rates of the wild type strain, three constructed deletion mutants, as well as the antibiotic resistant mutants obtained from the $\mathrm{Rif}^{\mathrm{R}}$ assay, were measured.

\section{Results}

Phenotypic characterization of the mutS, mutL single deletion mutants and the mutS mutL double deletion mutant

To identify the role of mutS and mutL in A. baylyi, we constructed a mutS mutL double deletion mutant (XH441) based on the mutL single deletion mutant XH440. A PCR fragment containing the cat gene which conferred chloramphenicol resistance and flanked by the regions surrounding the mutS gene, was introduced into XH440 by natural transformation (Fig. 1a). The A. baylyi mutS mutL double deletion mutant was obtained after selection on a chloramphenicol-containing plate. A specific PCR was used to confirm that the mutS gene was replaced by the cat gene, with subsequent sequencing of the fragment (Fig. 1b).

A.
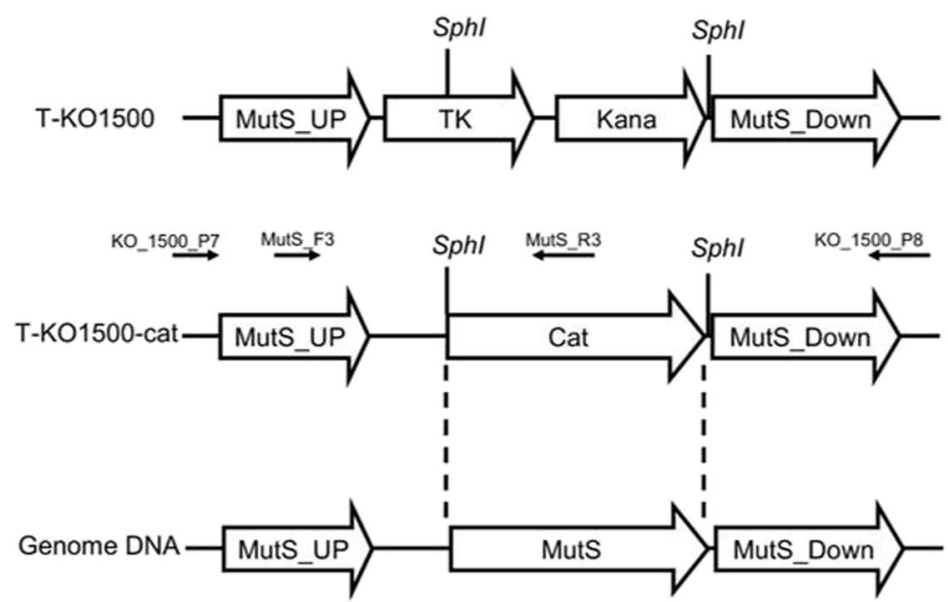

B.
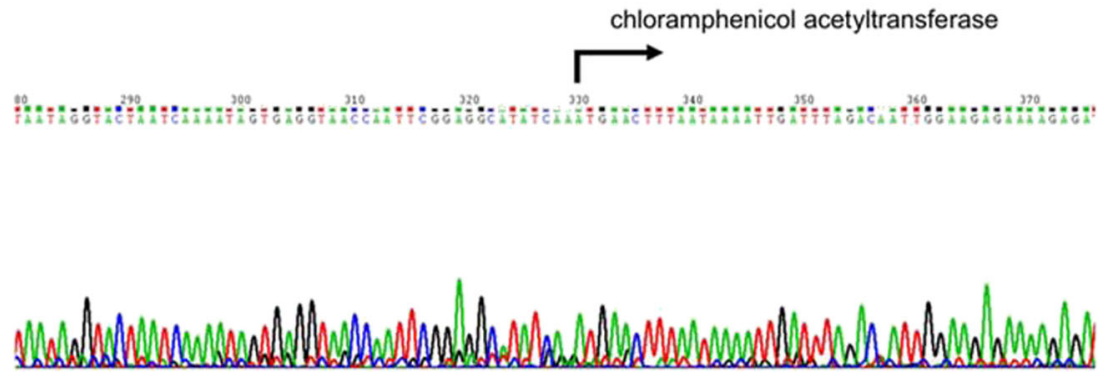

Fig. 1 knockout of mutS based on mutL single deletion mutant. a Schematic representation of the targeting strategy for generating a mutS mutL double deletion mutant. b Fluorescent dye chemistry sequencing was performed using the same primers used for PCR. The arrow suggested the start site of cat gene 


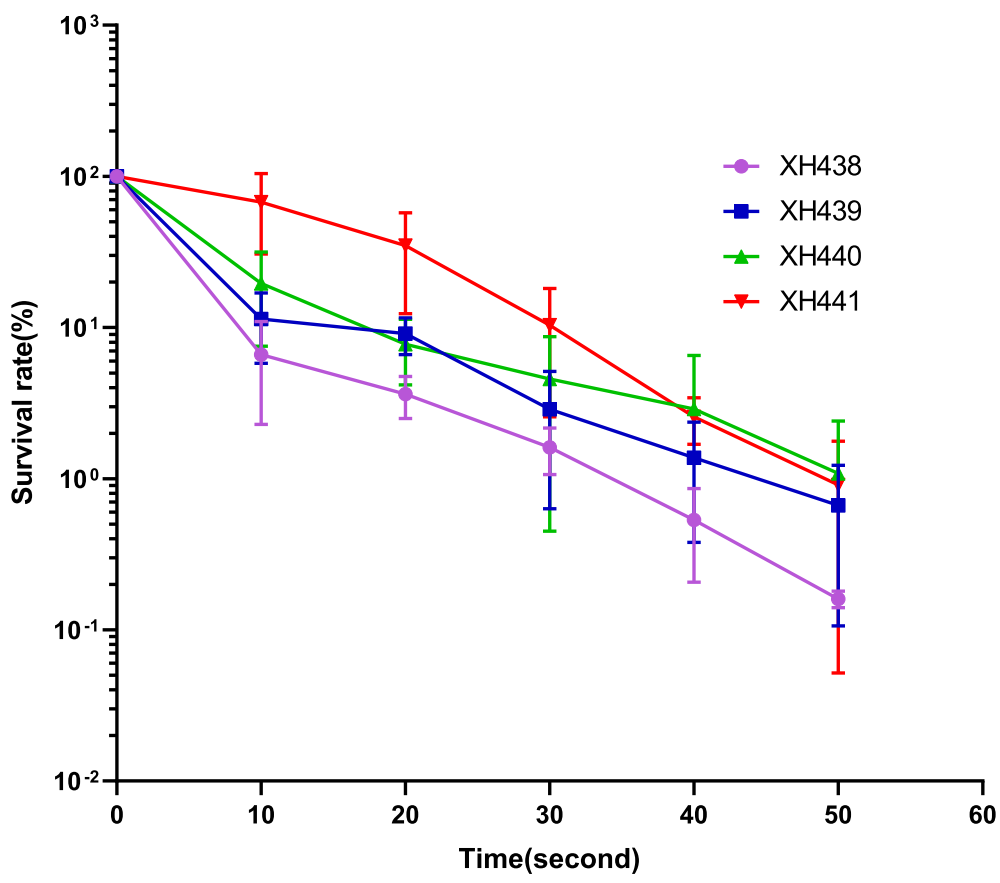

Fig. 2 UV sensitivity of A. baylyi ADP1 (XH438) and various mutants. XH439 (mutS), XH440 (mutL), XH441 (mutS mutL). The data are means of three independent experiments, error bars represent standard deviation

After obtaining XH441, we determined the cell survival rates after UV irradiation of the single and double deletion mutants and compared the rates with that of wild-type A. baylyi ADP1. As shown in Fig. 2, all mutants showed higher survival rates than wild type, especially the double mutants. These results could implicate that an alternate repair pathway might be employed when the MMR system is non-functional.

\section{Mutation frequencies and mutation rates in Rif ${ }^{\mathrm{R}}$ assay}

The mutation frequencies and mutation rates of wildtype and mutant $A$. baylyi strains were determined in the $\operatorname{Rif}^{\mathrm{R}}$ assay. Mutation frequencies of spontaneous occurrences of resistance towards rifampicin were determined and shown in Table 1. In the Rif ${ }^{\mathrm{R}}$ assay, all three mutS/mutL single and double mutants showed significantly higher frequencies than wild type. Inactivation of mutS conferred a 4-fold increase $\left(1.26 \times 10^{-8} / 3.02 \times 10^{-}\right.$ $\left.{ }^{9}\right)$ in the frequency of spontaneous rifampicin resistance compared to that of the wild type, whereas the mutation frequency of the mutL single mutant was 2 -fold that of wild type $\left(5.21 \times 10^{-9} / 3.02 \times 10^{-9}\right)$. XH441 generated a mutation frequency 11 -fold higher than that of wild type $\left(3.42 \times 10^{-8} / 3.02 \times 10^{-9}\right)$.

For Rif ${ }^{\mathrm{R}}$ assay, the mutation rate of the wild type strain ADP1 was estimated to be $1.6 \times 10^{-8}$ (95\% confidence interval, $7.7 \times 10^{-9}$ to $3.0 \times 10^{-8}$ ) based on the number of derived Rif ${ }^{\mathrm{R}}$ mutants. XH440 showed a similar mutation rate as the wild type (ADP1) $(p=0.802)$. Meanwhile,
XH439 displayed an approximately2-fold elevated rate compared with the rate for ADP1, but these rates were not significantly different $(p=0.116)$. Only XH441 showed a 4-fold increased mutation rate compared with ADP1 $(p<0.01)($ Table 1$)$.

\section{Mutation rates based on experimental evolution with whole-genome sequencing}

We performed laboratory evolution experiment with the wild type and its derivative deletion mutants (XH439, $\mathrm{XH} 440, \mathrm{XH} 441)$ to determine their mutant rates under no antibiotic pressure. The mutation rates of four strains were shown in Table 2 and we obtained a similar increasing trend of mutation rates in the previous $\mathrm{Rif}^{\mathrm{R}}$ assay except XH440 which showed a higher mutation rate in experimental evolution but a similar mutation

Table 1 Mutation frequencies and rates of XH438 (ADP1) and its deletion mutants in Rif ${ }^{\mathrm{R}}$ assay

\begin{tabular}{|c|c|c|c|}
\hline Strain & Genotype & $\begin{array}{l}\text { Mutation } \\
\text { frequency }\end{array}$ & Mutation rate \\
\hline XH438 & wild type & $3.02 \times 10^{-9}$ & $\begin{array}{l}1.64 \times 10^{-8}\left(7.68 \times 10^{-9}-\right. \\
\left.2.98 \times 10^{-8}\right)\end{array}$ \\
\hline $\mathrm{XH} 439$ & $\Delta m u t s$ & $1.26 \times 10^{-8}$ & $\begin{array}{l}3.02 \times 10^{-8}\left(1.88 \times 10^{-9}-\right. \\
\left.4.39 \times 10^{-8}\right)\end{array}$ \\
\hline $\mathrm{XH} 440$ & $\Delta$ mutl & $5.21 \times 10^{-9}$ & $\begin{array}{l}1.49 \times 10^{-8}\left(1.02 \times 10^{-8}-\right. \\
\left.2.06 \times 10^{-8}\right)\end{array}$ \\
\hline $\mathrm{XH} 441$ & $\Delta$ mutS $\Delta$ mutl & $3.42 \times 10^{-8}$ & $\begin{array}{l}6.44 \times 10^{-8}\left(4.15 \times 10^{-9}-\right. \\
\left.9.09 \times 10^{-8}\right)\end{array}$ \\
\hline
\end{tabular}


Table 2 Mutation rates of XH438 (ADP1) and its deletion mutants in experimental evolution

\begin{tabular}{|c|c|c|c|c|c|c|c|c|c|c|}
\hline Strain & Lines & $\begin{array}{l}\text { transfer } \\
\text { days }\end{array}$ & Generations & Ts & TV & Indel & $\begin{array}{l}\text { Point Mutation rate per } \\
\text { nucleotide }\left(\mu_{M A}\right)\end{array}$ & $\begin{array}{l}95 \% \text { confidence } \\
\text { interval }\end{array}$ & $\begin{array}{l}\text { Total Mutation rate per } \\
\text { nucleotide }\left(\mu_{M A}\right)\end{array}$ & $\begin{array}{l}95 \% \text { confidence } \\
\text { interval }\end{array}$ \\
\hline $\mathrm{XH} 438$ & 2 & 14 & 93 & 0 & 2 & 2 & $2.99 \times 10^{-9}$ & $\begin{array}{l}1.94 \times 10^{-10} \\
4.16 \times 10^{-9}\end{array}$ & $5.97 \times 10^{-9}$ & $\begin{array}{l}2.20 \times 10^{-9} \\
1.30 \times 10^{-8}\end{array}$ \\
\hline XH439 & 1 & 14 & 93 & 8 & 1 & 4 & $2.69 \times 10^{-8}$ & $\begin{array}{l}1.78 \times 10^{-8} \\
3.92 \times 10^{-8}\end{array}$ & $3.88 \times 10^{-8}$ & $\begin{array}{l}3.50 \times 10^{-8} \\
4.28 \times 10^{-8}\end{array}$ \\
\hline $\mathrm{XH} 440$ & 1 & 14 & 93 & 5 & 0 & 6 & $1.49 \times 10^{-8}$ & $\begin{array}{l}8.39 \times 10^{-9} \\
2.47 \times 10^{-8}\end{array}$ & $3.28 \times 10^{-8}$ & $\begin{array}{l}2.27 \times 10^{-8} \\
4.63 \times 10^{-8}\end{array}$ \\
\hline $\mathrm{XH} 441$ & 2 & 14 & 93 & 15 & 0 & 8 & $2.24 \times 10^{-8}$ & $\begin{array}{l}1.95 \times 10^{-8} \\
2.55 \times 10^{-8}\end{array}$ & $2.24 \times 10^{-8}$ & $\begin{array}{l}1.37 \times 10^{-8} \\
3.33 \times 10^{-8}\end{array}$ \\
\hline
\end{tabular}

Ts transition, Tv transversion, Indel insertion and deletion

rate in $\mathrm{Rif}^{\mathrm{R}}$ assay compared with wild-type strain. The mutation rate of the wild type strain ADP1 was estimated as $2.99 \times 10^{-9}$ (95\% confidence interval, 1.94 $\times$ $10^{-10}-4.16 \times 10^{-9}$ ) based on WGS analysis. All three mutants showed significantly higher point mutations rates than the wild type strain (5- to 9-fold).

\section{Mutational spectrums in Rif $^{\mathrm{R}}$ assay and experimental evolution}

To understand the mechanism of mutation, we isolated rifampicin resistant mutants derived from wild-type, XH439, XH440 and XH441 in Rif $^{\mathrm{R}}$ assay and sequenced $r p o B$ region which was related with $\mathrm{Rif}^{\mathrm{R}}$. Table 3 showed 225 mutations leading to the $\operatorname{Rif}^{R}$ phenotype, including 58 mutations occurring in wild type, 77 mutations in $\mathrm{XH} 439,68$ mutations in $\mathrm{XH} 440$ and 78 mutations in
XH441. The proportion of transition in ADP1 was $71.4 \%$ (40 of 56). The transition mutations were favored in three mutants (Fig. 3). XH439 favored three transition sites at position 1619, 1562, and 1604. Only one prominent transition site, position 1619, remained in XH440. XH441 not only maintained one transition site at position 1619 but also showed two other transition sites at positions 1573 and 1574. Knockout of mutL or mutS increased the proportion of $\mathrm{AT}=>\mathrm{GC}$ in transition mutations (Fig. 3).

A similar pattern of mutations' distribution was observed in experimental evolution. The sequence analysis of the final population evolved from wild type and its derivative deletion mutants (XH439, XH440, XH441) revealed a total of 4,13, 11 and 23 mutations, respectively (Table 2, Table S1). Base substitutions were two-fold

Table 3 Distribution of mutations leading to Rif $^{R}$ in $r p o B$

\begin{tabular}{|c|c|c|c|c|c|c|}
\hline $\begin{array}{l}\text { A.baylyi } \\
\text { Site (bp) }\end{array}$ & Amino acid change & Base-pair change & $\mathrm{XH} 438$ & XH439 & $\mathrm{XH} 440$ & $\mathrm{XH} 441$ \\
\hline 1561 & S521P & $\mathrm{AT}=>\mathrm{GC}$ & 2 & 0 & 1 & 6 \\
\hline 1565 & Q522R & $\mathrm{AT}=>\mathrm{GC}$ & 0 & 4 & 1 & 1 \\
\hline 1574 & D525G & $A T=>G C$ & 0 & 0 & 0 & 11 \\
\hline 1604 & H535R & $\mathrm{AT}=>\mathrm{GC}$ & 0 & 9 & 6 & 2 \\
\hline 1625 & L542S & $A T=>G C$ & 0 & 1 & 0 & 2 \\
\hline 1562 & S521F & $C G=>T A$ & 0 & 10 & 5 & 5 \\
\hline 1573 & D525N & $\mathrm{CG}=>\mathrm{TA}$ & 0 & 4 & 3 & 11 \\
\hline 1592 & S531F & $C G=>T A$ & 4 & 2 & 0 & 0 \\
\hline 1603 & $\mathrm{H} 535 \mathrm{Y}$ & $C G=>T A$ & 6 & 1 & 5 & 4 \\
\hline 1613 & $\mathrm{R} 538 \mathrm{H}$ & $C G=>T A$ & 0 & 1 & 1 & 3 \\
\hline 1619 & S540L & $\mathrm{CG}=>\mathrm{TA}$ & 11 & 28 & 48 & 27 \\
\hline 1718 & P573L & $C G=>T A$ & 17 & 7 & 7 & 5 \\
\hline 1564 & Q522K & $C G=>A T$ & 1 & 1 & 0 & 0 \\
\hline 1603 & H535N & $C G=>A T$ & 12 & 0 & 0 & 0 \\
\hline 1741 & $1581 \mathrm{~F}$ & $\mathrm{AT}=>\mathrm{TA}$ & 0 & 0 & 0 & 1 \\
\hline 1604 & H535P & $A T=>C G$ & 3 & 0 & 0 & 0 \\
\hline 1569 & 12 bp insertion & & 2 & 0 & 0 & 0 \\
\hline Total & & & 58 & 68 & 77 & 78 \\
\hline
\end{tabular}




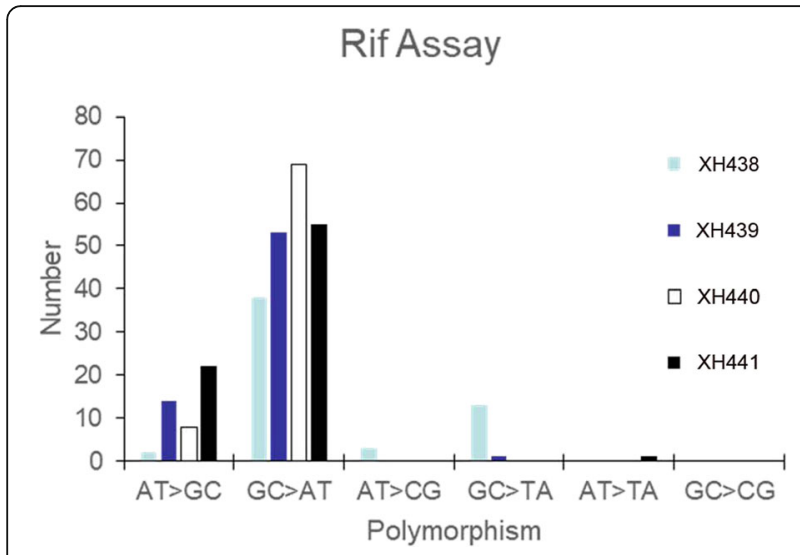

Fig. 3 Comparison of percentage of transitions and transversions in $r p o B$ in $A$. baylyi wild type and mutants in Rif $^{R}$ assay

more common than insertions/deletions in XH439 and XH441 (Table 2), and transitions were more abundant than transversions in all mutants (Fig. 4). The mutations seemed randomly distributed throughout the A. baylyi genome (Table S1). During our study, we identified several genes as mutation hotspots. For example, all six strains harbored a mutation in intergenic region between ACIAD_RS11450 and ACIAD_RS11455 (divalent metal cation transporter/LysR family transcriptional regulator). In addition, mutations in ACIAD_RS05875 (universal stress protein) appeared in five strains (5/6). Further research is required to understand the roles of these mutations in A. baylyi.

Effect of the mutS and mutL mutations on variations in marker replacement frequencies during transformation To detect the effect of mutS and mutL on the recognition of different mismatches in vitro, we transformed

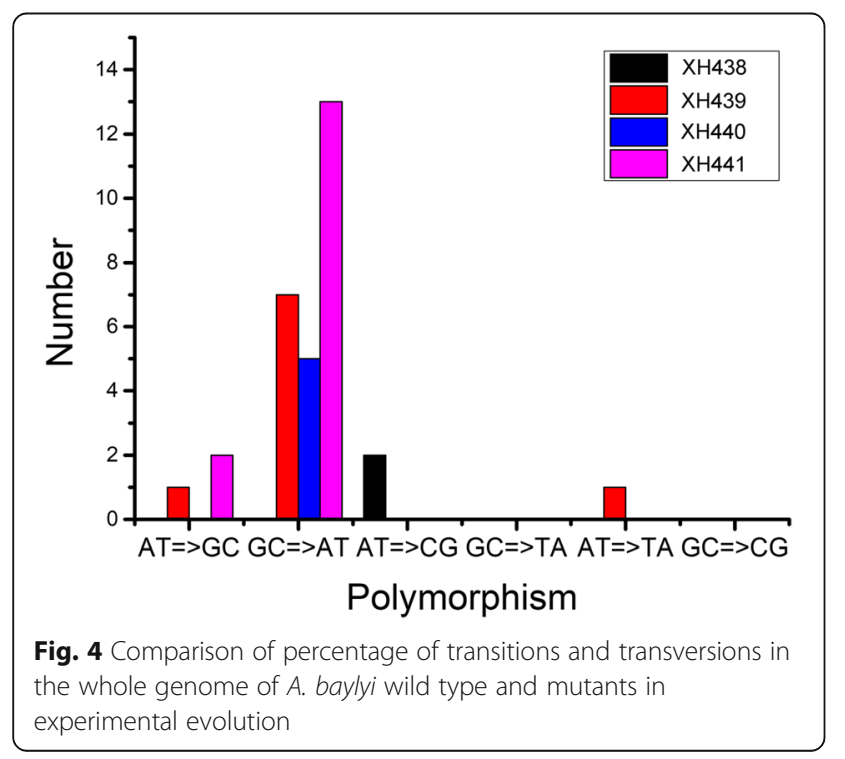

cells (wild-type, XH439, XH440, XH441) with genomic DNAs extracted from various spontaneous mutants obtained from Rif $^{\mathrm{R}}$ assay with a single point mutation (two transitions, three transversions) or $12 \mathrm{bp}$ insertion in rpoB that resulted in Rif $^{\mathrm{R}}$ phenotype. The transformation frequencies of $\mathrm{XH} 439$ were 0.95 - to 5.2-fold higher than those of the wild type $(p=0.33)$ (Fig. 5). XH440 also showed enhanced the transformation efficiency (0.97- to 3.5 -fold $)(p=0.30)$. XH441 generated a higher transformation efficiency of DNA than single-gene deletion mutants (1.52 to 5.4 -fold) $(p=0.02)$. No significant difference was detected in the interaction of receipt strain and donor DNA. Then, we divided the donor DNA into different mutation type, e.g. transition and transversion. XH441 showed a higher transformation efficiency than XH438 when donor DNA harbored transition mutations $(p<0.05)$.

\section{Effect of $r p o B$ mutations on the relative growth rate}

To investigate whether the $r p o B$ mutation affected the growth of $A$. baylyi, we determined the relative growth rate of wild type, its derivative mutant, and their iif $^{\mathrm{R}}$ mutants. XH439, XH440 and XH441 all showed higher growth rates than wild-type XH438 (Fig. 6a). For Rif ${ }^{R}$ mutants, $\mathrm{XH} 438$ displayed higher growth rate than its rpoB mutants, except H535N and S521P. XH440 grew faster than its rpoB mutants, except D525N. XH439 and XH441 showed significant higher growth rate than their rрoB mutants $(p<0.05)$. The growth rates showed that different types of rpoB mutations conferred variable fitness costs to $A$. baylyi. Interestingly, the effect of the $r p o B$ mutation on the growth rate was dependent on the mutation. We combined the growth rate data from different strains harboring different mutations, and compared them in pair.

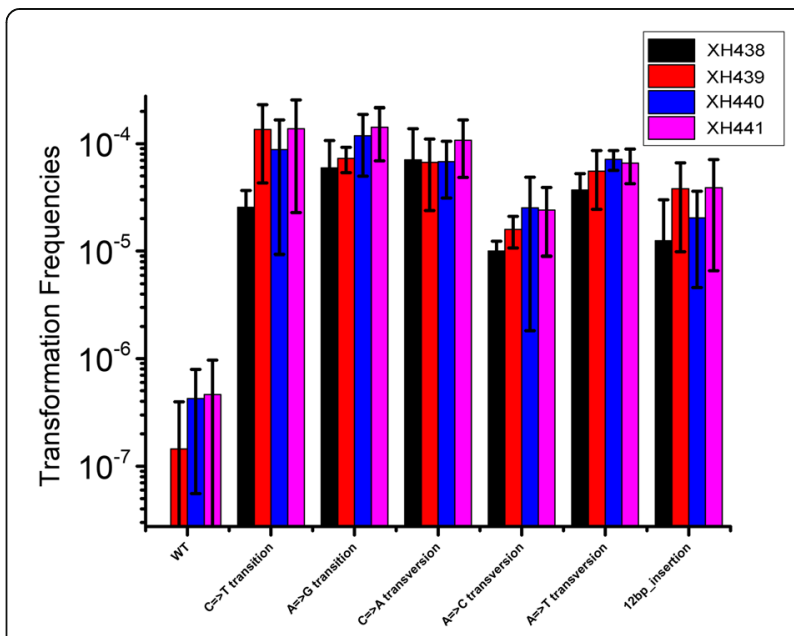

Fig. 5 Transformation frequencies of donor DNA containing rpoB mutation. The transformation frequency was measured as transformants per CFU under normal growth condition 


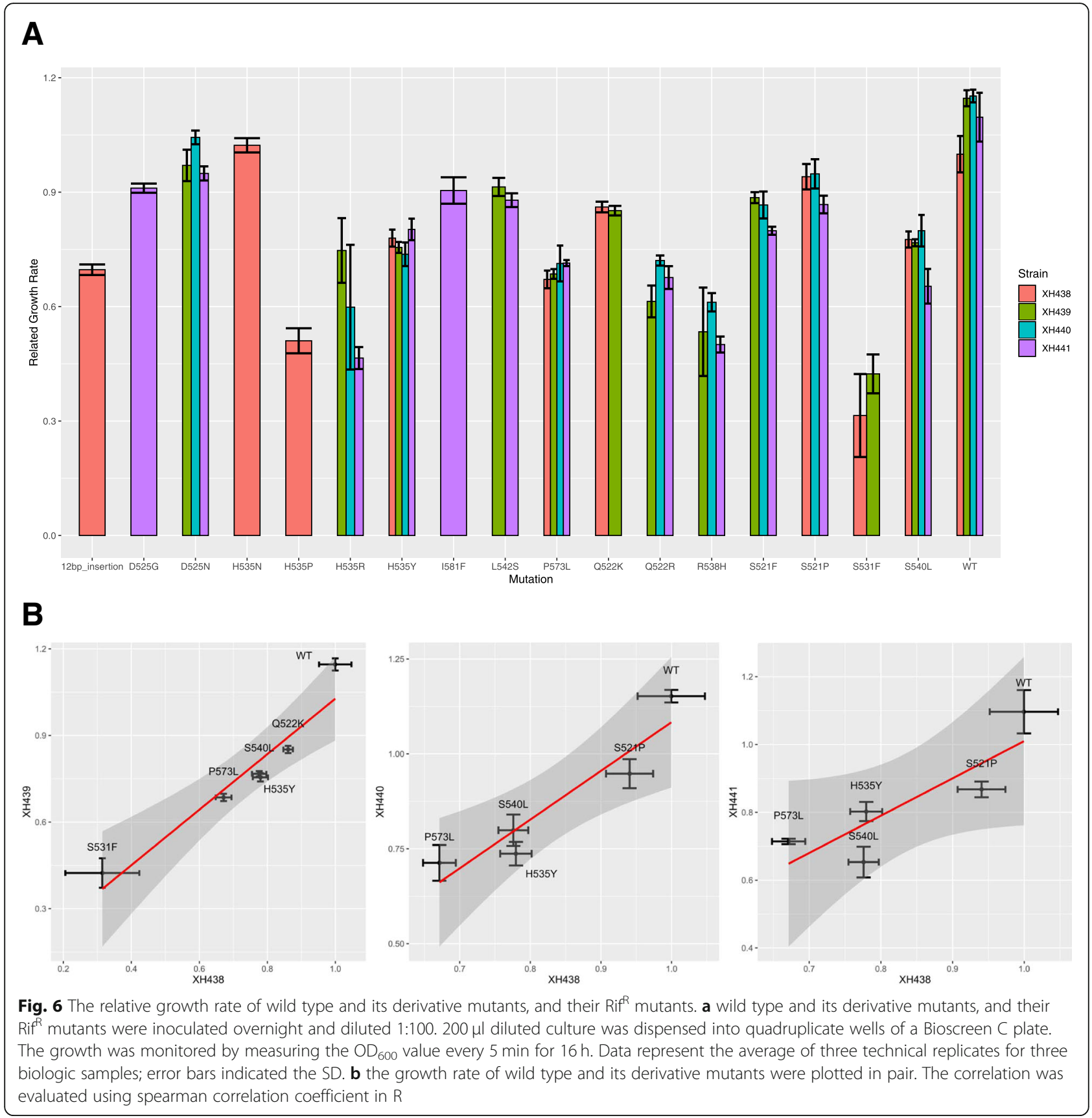

There was significant correlation between XH438 and its three deletion mutants (XH439, XH440, XH441) in growth rate (Fig. 6b). The spearman correlation coefficient between XH438 and its three deletion mutants are 0.94, 0.9 and 0.9 , respectively.

\section{Discussion}

In this study, we constructed a mutS mutL deletion mutant (XH441) and determined the mutation frequencies and rates in ADP1 (XH438), XH439, XH440 and XH441. XH439 (AmutS) showed a higher mutation frequency and mutation rate than XH440 ( $\Delta m u t L)$, which had a similar mutation rate and a slightly higher mutation frequency than the wild-type in Rif ${ }^{\mathrm{R}}$ assay. Although mutL was quite important to MMR, as it was reported to act not only as a matchmaker, but also provide endonuclease activity for strand incision in B. subtilis [15]. According to out result, it seemed that mutL could not repair the mismatches without mutS, which resulted in higher mutation frequency and mutation rate in XH439 ( $\Delta$ mutS). For the similar mutation rate and a slightly higher mutation frequency in XH440 ( $\Delta m u t L)$, 
we assumed that $\mathrm{Rif}^{\mathrm{R}}$ mutants derived from $\mathrm{XH} 440$ might have no relationship with mismatch repair as MutS was reported to affect RecA-mediated DNA strand exchange independently of MutL both in E. coli [16] and B. subtilis [17]. But, in the experimental evolution, the mutation rate in XH440 was 5.5-fold higher than the wild-type. It might because that half of the mutations detected in XH440 using WGS were Indels which were not able to be detected in Rif $^{R}$ assay (Table 2) (Table S1) as they did not occur on rpoB.

Mutations derived in a mismatch repair-deficient background are known to be predominantly $\mathrm{GC}=>\mathrm{AT}$ or $\mathrm{AT}=>\mathrm{GC}$ transitions $[18,19]$. In our study, Rif $^{\mathrm{R}}$ assay and experimental evolution confirmed that the major mutations occurred on either $r p o B$ or the whole genome were transition mutations. The increased proportion of $\mathrm{AT}=>\mathrm{GC}$ transition mutations was more common in mutant strains. In Rif ${ }^{\mathrm{R}}$ assay, we were able to detect mutations occurred on $r р о B$ only. While the experimental evolution and the following WGS enabled us to detect additional types of mutations, e.g., short insertions or deletions and large deletions. The combination of experimental evolution and whole-genome sequencing provides an improved understanding of mutation formation in A. baylyi.

The MMR system was originally described in S. pneumoniae $[8,9]$. At first, the proteins involved in the system were termed HexA and HexB. Previous study reported that the loss of mutS increased the transformation frequencies for the transition but did not affect the transformation frequencies of transversions in $A$. baylyi [14]. Our results showed that there are no significantly differences between the transformation frequencies of transition, transversion and insertion in four strains. Only XH441 showed a higher transformation efficiency than XH438 when donor DNA harbored transition mutations. The result confirmed that transition was favored in mutSL mutants.

Antibiotic resistance based on genetic mutations on chromosomes is often accompanied by fitness costs. Drug-resistant mutations in $r p o B$ were mostly located in the 81-bp region of rроB (rifampicin resistance determining region, RRDR). These rpoB mutations often come with fitness costs [20]. In this study, we reported the fitness cost of 14 types of $r p o B$ base substitution and one type of 12-bp insertion. Most of rpoB mutation in four strains confirmed fitness cost. The growth rates showed that different types of rpoB mutations conferred variable fitness costs to $A$. baylyi and that there was also a large difference in the fitness of different missense mutations at the same amino acid position. The influence of rpo $B$ mutations have been proved to be rather complex under different conditions in several studies. $r р о B$ mutation in Clostridium difficile was associated with fitness cost in vitro and reduced virulence in vivo [21]. Mutations at position 522 or 540 in rpoB of A. baumannii were impaired in surface-associated motility and showed attenuated virulence [22]. The four different $r p o B$ mutations in $E$. coli exhibited deleterious fitness costs under nutrient-rich conditions, but some rpoB alleles showed a remarkable fitness increase under phosphate limitation conditions [23]. In S. aureus, $\mathrm{Rif}^{\mathrm{R}}$ mutants exhibited increased growth within biofilms [24]. The difference suggested that bacterial microenvironments should be considered before general conclusions on fitness cost are drawn [23]. To our knowledge, our study provides the first evaluation of the fitness cost associated with $A$. baylyi rifampin resistance in vitro.

\section{Conclusions}

We demonstrated that inactivation of both mutS and mut $L$ increased the mutation rates and frequencies in $A$. baylyi, which would contribute to the evolution and acquirement of rifampicin resistance. The mutS single deletion is also implicated in increased mutation rates and frequencies, suggesting that MutL may be activated even in the absence of mutS. The correlation between fitness cost and rifampin resistance mutations in A. baylyi is firstly established.

\section{Methods}

\section{Bacterial strains, media and antibiotics}

Restriction enzymes, T4 ligase, and Taq DNA polymerase were purchased from TaKaRa (Otsu, Shiga, Japan). All A. baylyi cultures (Table 4) were grown at $37^{\circ} \mathrm{C}$ in LB broth and agar (Oxoid, Basingstoke, UK). Rifampin (Rif), kanamycin and chloramphenicol were purchased from Sangon (Shanghai, China) and dissolved in methanol.

\section{Construction of $A$. baylyi mutS mutL mutant}

A. baylyi ADP1, XH439, XH439 and XH440 were provided by the Commissariat à l'Energie Atomique/Direction des Sciences du Vivant [2]. We constructed a mutS

Table 4 Bacterial strains and plasmids used in the study

\begin{tabular}{lll}
\hline Strain/ plasmid & Relevant characteristic(s) & Source \\
\hline XH438 & A.baylyi ADP1, Wild type & CEA \\
XH439 & As ADP1 but mutS::kana & CEA \\
XH440 & As ADP1 but mutL::kana & CEA \\
XH441 & As KO2375 but mutS::cat & This study \\
T-KO1500 & PCR fragment of flanking & This study \\
& $\begin{array}{l}\text { regions of kana which } \\
\text { replaced mutS in XH440 }\end{array}$ & \\
& Cloned into T-vector, & \\
& Ampr', Kanar & \\
T-KO1500-cat & PCR fragment of cat & This study \\
& gene cloned into T-KO1500 & \\
\hline
\end{tabular}


mutL double mutant based on XH440. A DNA fragment was amplified from the XH439 mutant by primers $\mathrm{KO}_{-}$ 1500_P7 and KO_1500_P8 and ligated into the pMD 18$\mathrm{T}$ vector as T-XH439. The cat gene was amplified from pTEX5500ts by cat_F2 and cat_R2, digested with SphI, and subcloned into T-XH439 as T-XH439-cat. The PCR fragment used to knockout mutS was amplified from T$\mathrm{XH} 439$-cat and transformed into $\mathrm{XH} 440$, and the mutant was selected on a chloramphenicol-containing plate. The double mutant was verified by PCR and DNA sequencing.

Survival rates characterization of $A$. baylyi and its mutants UV irradiation experiment was conducted as described by Thoms and Wackernagel [25] with minor modifications. Bacteria overnight cultures in LB broth with a density of $10^{8}$ cells $/ \mathrm{ml}$ were centrifuged and resuspended in $1 \times$ phosphate buffer (Senrui, China). Cells were irradiated at room temperature with a UV lamp in a biological safety cabinet (Thermo Scientific 1300 Series B2) at a distance of $60 \mathrm{~cm}$. The cell suspension had a depth of less than $1 \mathrm{~mm}$. Before and after irradiation, samples were withdrawn to estimate the survival rate of bacteria. Samples were plated on agar plates after appropriate dilution and colonies were counted after about 24 $\mathrm{h}$ incubation at $37^{\circ} \mathrm{C}$. The experiment was performed three times independently. Survival rates (\%) were presented as the mean \pm SD (standard deviation).

\section{Measurement of mutation frequency and mutation rate in Rif $^{\mathrm{R}}$ assay}

For mutation frequency assays, overnight cultured cells were harvested, washed twice with phosphate-buffered saline (PBS) solution and re-suspended in PBS. Serial dilutions with PBS were plated onto LB plates containing $50 \mu \mathrm{g} / \mathrm{mL}$ Rif and incubated for $24 \mathrm{~h}$ before scoring. The total number of colony-forming units (CFU) was determined by plating serial dilutions on LB plates. The frequencies of mutations conferring resistance to Rif were determined by dividing the median number of mutants by the average number of cells [26].

For mutation rates, the maximum-likelihood estimator applying the newton. LD.plating function from the rSalvador package v1.7 for $\mathrm{R}$ was used to estimate the mutation rate $(\mu)$ to $\mathrm{Rif}^{\mathrm{R}}$ in each strain, and statistical comparisons were performed by using the likelihood ratio test (LRT.LD.plating function from rSalvador) [27]. The colonies in the Rif ${ }^{\mathrm{R}} \mathrm{LB}$ plates were used for genomic DNA isolation, PCR, and Sanger sequencing.

\section{DNA isolation and $r p o B$ sequencing of Rif $^{\mathrm{R}}$ mutants}

Genomic DNA was isolated from the colonies in the Rif $^{R}$ LB plates using a TianGen genome isolation kit (TianGen Biotech Company Ltd., Beijing, China). The primers
ADP_rpoB_1S2 and ADP_rpoB_1A (Table 5) were used to amplify the DNA for sequencing. The PCR products were purified and sequenced by Biosune biological company (Hangzhou, China). The sequences and trace data were transferred to the SEQMAN program (DNASTAR), which was used for sequence assembly and SNP detection [26].

\section{Experimental evolution and measurement of mutation rate in A. baylyi ADP1 and its derivative mutants}

A single colony of $A$. baylyi ADP1 and its derivative mutants were cultured in $2 \mathrm{~mL}$ of $\mathrm{LB}$ broth overnight at $37^{\circ} \mathrm{C}$. Then, the bacteria were serially passaged for 14 days. The final cultures were stored at $-80^{\circ} \mathrm{C}$. The genomic DNA was extracted and sequenced as previously described [28]. Briefly, the genomic DNA was extracted using a QIAamp DNA Mini Kit (Qiagen Valencia, CA). The genome was sequenced on an Illumina HiSeq platform (Illumina, San Diego, CA). Mutations in evolutionary strains were identified by Breseq [29]. The mutation rate was calculated via equation $\mu \mathrm{MA}=\frac{m}{\sum_{i=1}^{n} N_{i} \times \mathrm{T}_{i}}$, where $m$ is the total number of mutations in all strains, $n$ is the number of lines, $N_{i}$ is the number of nucleotide sites, and $T$ is the number of generations of bacteria during passage. Confidence intervals were calculated from a Poisson distribution using Poisson's test in R [30].

\section{Natural transformation with genomic DNA with the rpoB mutation}

For transformation with genomic DNA with the $r p o B$ mutation, the genomic DNA were extracted from the known $r p o B$ mutation isolates (including transition, transversion, and insertion). DNA was quantified by a NanoDrop ND-1000 spectrophotometer. Overnight cultured cells diluted 1:100 to fresh $\mathrm{LB}$ broth at $37^{\circ} \mathrm{C}$ at $250 \mathrm{rpm}$ for $2 \mathrm{~h}$, and DNA was added to a final concentration of $400 \mathrm{ng} / \mathrm{mL}$. After $3 \mathrm{~h}$ at $37^{\circ} \mathrm{C}$, cells were plated on LB plates, and LB plates contained $50 \mu \mathrm{g} / \mathrm{mL}$ rifampin. The plates were incubated for $24 \mathrm{~h}$ before scoring. The transformation frequencies were determined as the

Table $\mathbf{5}$ primers used in the study

\begin{tabular}{ll}
\hline Primer name & Sequence(5'-> 3') \\
\hline KO_1500_P7 & CCCATCTTTCTACAAGTAACGCTTAAACC \\
KO_1500_P8 & CTAGACATTGGACAAAATAGCC \\
cat_F2 & GCATGCCGTAAAATTTGTTTGATTTTCC \\
cat_R2 & GCATGCTTTCATTAGTCCATTACCTGGT \\
ADP_rpoB_1S2 & TCGTTGCGGATACTTTGCGTGC \\
ADP_rpoB_1A & GCAAAGTTGGAACAGCCTGACG \\
MutS_F3 & AAGCGAGATGTCTGTAGAAGTT \\
MutS_R3 & GCTGTAATAATGGGTAGAAGGT \\
\hline
\end{tabular}


ratio of mutant bacteria on $\mathrm{Rif}^{\mathrm{R}}$ plates to total viable bacteria on LB plates [31]. Three independent biological replicates were performed. ANOVA with TukeyHSD posthoc test was performed to identify differences between each strain when different donor DNA provided [32].

\section{Measurement of growth rate of ADP1 and derivative mutants}

Three independent colonies per strain were grown in $\mathrm{MH}$ medium overnight and diluted to 1:100 in $\mathrm{MH}$ medium, and aliquots were placed into a flat-bottom 100 -well plate in three replicates. Then, the plate was incubated at $37{ }^{\circ} \mathrm{C}$, and the $\mathrm{OD}_{600}$ was detected every 5 min for $16 \mathrm{~h}$ using a Bioscreen C MBR machine (Oy Growth Curves Ab Ltd., Finland). The growth rate was estimated based on $\mathrm{OD}_{600}$ curves via BAT 2.0 [33]. The correlation of growth rate between different strains was evaluated using Spearman correlation coefficient in $\mathrm{R}$ [32]. The figures were plotted using ggplot2 [34].

\section{Supplementary information}

Supplementary information accompanies this paper at https://doi.org/10. 1186/s12866-020-01729-3.

Additional file 1 : Table S1. Predicted mutations in A. baylyi ADP1 from the WGS experiments.

\section{Abbreviations}

A. baylyi: Acinetobacter baylyi; CFU: Colony-forming units; MMR: Mismatch repair; PBS: Phosphate-buffered saline; Rif: Rifampin; RifR: Rifampin resistance; UV: Ultraviolet

\section{Acknowledgements}

We thank CEA/Direction des Sciences du Vivant for the mutS and mutL knockout strains of A. baylyi. We thank Prof. Sebastian Leptihn, Zhejiang University-University of Edinburgh Institute for critical reading of this manuscript. This manuscript has benefited greatly form the constructive comments of anonymous reviewers.

\section{Authors' contributions}

$\mathrm{HZ}$ analyzed experimental data and drafted the manuscript. LYZ constructed A. baylyi mutants, conducted the survival rates with UV, performed the measurement of mutation frequency and growth rate. QX and $\mathrm{LHZ}$ performed the experiment of natural transformation and experimental evolution. $\mathrm{XH}$ and $\mathrm{YY}$ designed the study and drafted the manuscript. All authors read and approved the final manuscript.

\section{Funding}

This work was supported by the National Natural Science Foundation of China (Grant No. 31970128, 31770142, 31670135), and the Zhejiang Province Medical Platform (2020RC075)

\section{Availability of data and materials}

The whole-genome shotgun projects of the A. baylyi strains have been deposited at DDBJ/EMBL/GenBank under the accession numbers MPVT00000000-MPVY00000000. The versions described in this paper are versions MPVT00000000-MPVY00000000.

Ethics approval and consent to participate Not applicable
Consent for publication

Not applicable.

\section{Competing interests}

The authors declare that they have no competing interests.

\section{Author details}

${ }^{1}$ Department of Respiratory Diseases, the First Affiliated Hospital, Zhejiang University School of Medicine, Hangzhou, China. ${ }^{2}$ Department of Infectious Diseases, Sir Run Run Shaw Hospital, Zhejiang University School of Medicine, Hangzhou, China. ${ }^{3}$ Key Laboratory of Microbial Technology and

Bioinformatics of Zhejiang Province, Hangzhou, China.

Received: 26 June 2019 Accepted: 14 February 2020

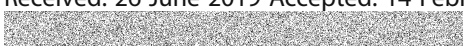

\section{References}

1. Hare JM, Perkins SN, Gregg-Jolly LA. A constitutively expressed, truncated umuDC operon regulates the recA-dependent DNA damage induction of a gene in Acinetobacter baylyi strain ADP1. Appl Environ Microbiol. 2006;72(6): 4036-43.

2. de Berardinis V, Vallenet D, Castelli V, Besnard M, Pinet A, Cruaud C, Samair S, Lechaplais C, Gyapay G, Richez C, et al. A complete collection of singlegene deletion mutants of Acinetobacter baylyi ADP1. Mol Syst Biol. 2008;4: 174.

3. Elliott KT, Neidle EL. Acinetobacter baylyi ADP1: transforming the choice of model organism. IUBMB Life. 2011;63(12):1075-80.

4. Overballe-Petersen S, Harms K, Orlando LA, Mayar JV, Rasmussen S, Dahl TW, Rosing MT, Poole AM, Sicheritz-Ponten T, Brunak S, et al. Bacterial natural transformation by highly fragmented and damaged DNA. Proc Natl Acad Sci U S A. 2013;110(49):19860-5.

5. Barbe V, Vallenet D, Fonknechten N, Kreimeyer A, Oztas S, Labarre L, Cruveiller S, Robert C, Duprat S, Wincker $\mathrm{P}$, et al. Unique features revealed by the genome sequence of Acinetobacter sp. ADP1, a versatile and naturally transformation competent bacterium. Nucleic Acids Res. 2004; 32(19):5766-79.

6. Busch CR, DiRuggiero J. MutS and MutL are dispensable for maintenance of the genomic mutation rate in the halophilic archaeon Halobacterium salinarum NRC-1. PLoS One. 2010;5(2):e9045

7. Willems RJ, Top J, Smith DJ, Roper DI, North SE, Woodford N. Mutations in the DNA mismatch repair proteins MutS and MutL of oxazolidinoneresistant or -susceptible Enterococcus faecium. Antimicrob Agents Chemother. 2003;47(10):3061-6.

8. Ephrussi-Taylor H, Gray TC. Genetic studies of recombining DNA in pneumococcal transformation. J Gen Physiol. 1966;49(6):211-31.

9. Lacks S. Molecular fate of DNA in genetic transformation of pneumococcus. Jol Biol. 1962;5:119-31.

10. Li Y, Schroeder JW, Simmons LA, Biteen JS. Visualizing bacterial DNA replication and repair with molecular resolution. Curr Opin Microbiol. 2018; 43:38-45

11. Sachadyn P. Conservation and diversity of MutS proteins. Mutat Res. 2010; 694(1-2):20-30

12. Castaneda-Garcia A, Prieto Al, Rodriguez-Beltran J, Alonso N, Cantillon D, Costas C, Perez-Lago L, Zegeye ED, Herranz M, Plocinski P, et al. A noncanonical mismatch repair pathway in prokaryotes. Nat Commun. 2017:8: 14246.

13. Kamashev D, Oberto J, Serebryakova M, Gorbachev A, Zhukova Y, Levitskii S, Mazur AK, Govorun V. Mycoplasma gallisepticum produces a histone-like protein that recognizes base mismatches in DNA. Biochemistry. 2011;50(40): 8692-702.

14. Young DM, Ornston LN. Functions of the mismatch repair GenemutS from Acinetobacter sp. strain ADP1. J Bacteriol. 2001;183(23):6822-31.

15. Pillon MC, Lorenowicz JJ, Uckelmann M, Klocko AD, Mitchell RR, Chung YS, Modrich P, Walker GC, Simmons LA, Friedhoff $P$, et al. Structure of the endonuclease domain of MutL: unlicensed to cut. Mol Cell. 2010; 39(1):145-51.

16. Worth L Jr, Clark S, Radman M, Modrich P. Mismatch repair proteins MutS and MutL inhibit RecA-catalyzed strand transfer between diverged DNAs. Proc Natl Acad Sci U S A. 1994;91(8):3238-41. 
17. Carrasco B, Serrano E, Martin-Gonzalez A, Moreno-Herrero F, Alonso JC. Bacillus subtilis MutS modulates RecA-mediated DNA Strand exchange between divergent DNA sequences. Front Microbiol. 2019;10:237.

18. Schaaper RM, Dunn RL. Spectra of spontaneous mutations in Escherichia coli strains defective in mismatch correction: the nature of in vivo DNA replication errors. Proc Natl Acad Sci U S A. 1987:84(17):6220-4.

19. Leong PM, Hsia HC, Miller JH. Analysis of spontaneous base substitutions generated in mismatch-repair-deficient strains of Escherichia coli. J Bacteriol. 1986;168(1):412-6.

20. Munir A, Kumar N, Ramalingam SB, Tamilzhalagan S, Shanmugam SK, Palaniappan AN, Nair D, Priyadarshini P, Natarajan M, Tripathy S, et al. Identification and characterization of genetic determinants of isoniazid and rifampicin resistance in Mycobacterium tuberculosis in southern India. Sci Rep. 2019;9(1):10283.

21. Kuehne SA, Dempster AW, Collery MM, Joshi N, Jowett J, Kelly ML, Cave R, Longshaw CM, Minton NP. Characterization of the impact of rpoB mutations on the in vitro and in vivo competitive fitness of Clostridium difficile and susceptibility to fidaxomicin. J Antimicrob Chemother. 2018;73(4):973-80.

22. Perez-Varela M, Corral J, Vallejo JA, Rumbo-Feal S, Bou G, Aranda J, Barbe J. Mutations in the beta-subunit of the RNA polymerase impair the surfaceassociated motility and virulence of Acinetobacter baumannii. Infect Immun. 2017;85(8):e00327-17.

23. Maharjan R, Ferenci T. The fitness costs and benefits of antibiotic resistance in drug-free microenvironments encountered in the human body. Environ Microbiol Rep. 2017;9(5):635-41.

24. Maudsdotter L, Ushijima Y, Morikawa K. Fitness of spontaneous rifampicinresistant Staphylococcus aureus isolates in a biofilm environment. Front Microbiol. 2019;10:988.

25. Thoms B, Wackernagel W. UV-induced allevation of lambda restriction in Escherichia coli K-12: kinetics of induction and specificity of this SOS function. Mol Gen Genet. 1982;186(1):111-7.

26. Hua X, Xu X, Li M, Wang C, Tian B, Hua Y. Three nth homologs are all required for efficient repair of spontaneous DNA damage in Deinococcus radiodurans. Extremophiles. 2012;16(3):477-84.

27. Zheng Q. rSalvador: an $\mathrm{R}$ package for the fluctuation experiment. $\mathrm{G} 3$ (Bethesda). 2017;7(12):3849-56.

28. Xu Q, Chen T, Yan B, Zhang L, Pi B, Yang Y, Zhang L, Zhou Z, Ji S, Leptihn S, et al. Dual role of gnaA in antibiotic resistance and virulence in Acinetobacter baumannii. Antimicrob Agents Chemother. 2019;63(10): e00694-19. https://doi.org/10.1128/AAC.00694-19.

29. Deatherage DE, Barrick JE. Identification of mutations in laboratory-evolved microbes from next-generation sequencing data using breseq. Methods Mol Biol. 2014;1151:165-88

30. Szafranska AK, Junker V, Steglich M, Nubel U. Rapid cell division of Staphylococcus aureus during colonization of the human nose. BMC Genomics. 2019;20(1):229.

31. Kickstein E, Harms K, Wackernagel W. Deletions of recBCD or recD influence genetic transformation differently and are lethal together with a recJ deletion in Acinetobacter baylyi. Microbiology. 2007;153(Pt 7):2259-70.

32. Team RC. R: a language and environment for statistical computing. Vienna: R for Statistical Computing; 2019.

33. Thulin M. BAT v.2.0. Uppsala: Uppsala University; 2018.

34. Wickham H. ggplot2: elegant graphics for data analysis. New York: SpringerVerlag; 2016.

\section{Publisher's Note}

Springer Nature remains neutral with regard to jurisdictional claims in published maps and institutional affiliations.

\section{Ready to submit your research? Choose BMC and benefit from:}

- fast, convenient online submission

- thorough peer review by experienced researchers in your field

- rapid publication on acceptance

- support for research data, including large and complex data types

- gold Open Access which fosters wider collaboration and increased citations

- maximum visibility for your research: over $100 \mathrm{M}$ website views per year

At $\mathrm{BMC}$, research is always in progress.

Learn more biomedcentral.com/submissions 